\title{
How Personality Impacts Remote Workers in Mainland China: A Qualitative Study
}

\author{
Christopher Van Ham ${ }^{1}$ \\ Independent Researcher, USA
}

\begin{abstract}
The present study identifies and discusses the impact of personality factors on remote workers in China, using a phenomenological methodology to understand participants' lived experiences. The sample population is comprised of Chinese citizens born in China. Data were collected through one-on-one, online semi-structured interviews using WeChat. The interview questions are structured to allow participants to explain personality traits found in productive and unproductive remote workers. In addition, the questions explore traits positively associated with developing trust and how the psychological needs of autonomy, competency, and relatedness impact remote workers. After data collection, the data were analyzed and coded to develop themes used to answer the core research questions. This study's findings offer benefits to companies using remote workers and employees new to remote work or those aiming to become remote workers since the data collected provides a holistic picture of remote work from actual workers' perspective. The information gathered from the research participants allowed the researcher to determine that one of the most beneficial aspects of remote work is autonomy and the ability to set one's schedule. Confidence directly impacts feelings of competency among remote workers, and relatedness is negatively impacted by being a remote worker because of decreased opportunities for face-to-face interactions.
\end{abstract}

KEYWORDS: China, personality, remote work, self-determination theory, phenomenology.

As a result of the COVID-19 pandemic, numerous people experienced remote work for the first time; however, remote work is not a novel concept. Research on remote work began in the 1970s with investigations of how remote work could solve problems such as productivity loss, traffic congestion, pollution, consumption of fossil fuels, and urban sprawl (Nilles, 1997). Recently, numerous articles have focused on remote work. Unfortunately, many of these articles use similar concepts to draw conclusions, mainly focusing on the benefits and drawbacks of working remotely. Thus, a little additional benefit is available to exploring remote work through this prism. Specific benefits previously cited in some studies include flexibility, fewer distractions, autonomy (Smith et al., 2015), a worker's ability to work during their most productive time (Nakrošienè et al., 2019), a less stressful work environment, and lower employee turnover (Choi, 2019).

De Vos et al. (2018) posited remote work as a means of decreasing car travel, which expanded on the seminal work of Nilles (1991), where it was stated that an immediate effect of remote work is a reduction in air pollution and car congestion. Nilles's findings connect with the

\footnotetext{
${ }^{1}$ Corresponding Author: Doctoral researcher at California Southern University. E-Mail: chrisvanham@yahoo.com
} 
stated goals of the Chinese government. In the $14^{\text {th }}$ five-year economic plan of March 2021, the Chinese government stated their goal of reducing energy consumption by $13.5 \%$ and GDP carbon dioxide emissions per unit by $18 \%$ (The $14^{\text {th }}$ five-year plan for economic and social development of the People's Republic of China, 2021). Thus, remote work in China can help meet the government's environmental goals. In addition, remote work can decrease car travel which can help address traffic congestion in China's cities.

Self-determination theory states that all people possess three psychological needsautonomy, relatedness, and competency- which are at the core of healthy psychological functioning and well-being (Deci \& Ryan, 2000; Kim \& Beehr, 2020; Unanue et al., 2017). The source of these basic psychological needs is the self (autonomy), demonstrating a sense of closeness with others (relatedness), and having a sense of control (competency) (Kuem et al., 2020). As opposed to typical behavioral approaches, self-determination theory focuses on people's inherent motivational forces and how these motivational forces impact learning and growing as well as how to support people (Ryan \& Deci, 2020). Clarifying how these psychological needs impact remote workers is the core of research question three.

Self-determination theory illustrates that the satisfaction of psychological needs is linked to higher well-being at work and in life (Kim \& Beehr, 2020; Unanue et al., 2017). In other words, intrinsic and extrinsic factors play a central role in regulating the behavior of people (Kuem et al., 2020). Ensuring remote workers' needs are met is crucial because autonomy, relatedness, and competency impact people's psychological health.

There is a gap in the research surrounding remote work because cultural perceptions of people from China and individualistic countries are not appropriately addressed in current literature. This research addressed and filled-in this gap by looking at remote workers in mainland China. Specifically, the concept of remote work was researched, self-determination theory, Chinese cultural concepts, and individuals from China with remote work experience were interviewed.

\section{Background of the Problem}

The existing research on remote work mainly draws upon European or United States perspectives, concentrating on people from Western cultures; consequently, this research may not apply to people of other cultures. For example, Chinese culture is different from Western cultures. For companies, especially non-Chinese companies, to effectively employ remote workers in China, understanding what type of employees will thrive in remote work settings is critical.

People from different cultures tend to bring contrary beliefs, values, norms, and behaviors to their cross-cultural relationships (Jukka et al., 2017). Accordingly, this study contributed to and expanded upon existing research in several ways. First, this research study was conducted in mainland China, and the research population was comprised of people from China. The purpose of focusing on China is that the unique cultural aspects of Chinese society need to be understood in the context of remote work because of China's economic strength and growth. The Chinese government is embracing technological advancement and change. These changes offer potential benefits for benefit remote work in China. For example, the government's goal is to expand 5G networks and increase user penetration to $56 \%$ while beginning to lay out a $6 \mathrm{G}$ network (The 14 th five-year plan for economic and social development of the People's Republic of China, 2021).

Secondly, this study used a phenomenological approach in focusing solely on one group of

people. By interviewing people in China with remote work experience, this research started to uncover which personality traits are present in productive and unproductive remote workers. The research also explored how unique cultural aspects of China and self-determination theory impact remote workers. This analysis is critical because the research will help guide employees and 
employers in making better career and hiring decisions. The research also provides suggestions for future research related to remote work. Economically this research has significant implications for the hiring and employment practices of companies operating in China. On a larger scale, this research has pioneered the process of identifying personality traits for productive and unproductive remote workers in China.

In the study of remote work, personality traits and self-determination theory have not been adequately researched. The term personality trait requires context. A personality trait focuses on an individual's intelligence, temperament, self-expression, and sociality. Personality is a foundational characteristic that impacts and influence people's behavior (Potard et al., 2020).

This study focused on answering three distinct questions that previous research has not adequately addressed. These questions form the core of this research project, and all three questions shaped the semi-structured interview portion of the data collection process. The research questions are referenced during the data analysis and explanation.

Research Question 1: What personality traits are found in productive and unproductive remote workers?

Research Question 2: Which traits are more positively associated with developing trust? Research Question 3: What specific psychological needs such as autonomy, competency, and relatedness are associated with remote workers who have more than 18 months of remote work experience?

In their seminal work, Morgeson and Humphrey (2006) stated that task characteristics and how work is accomplished are the most widely studied motivational work design. However, their research stopped short of looking for personality traits that may impact an individual's ability to do their job successfully, and did not focus on remote work. It should be noted that there is likely variance between how individuals are affected by domain interference, such as at home and work (Chong et al., 2018).

By fully understanding the personality make-up that accommodates workers' successful migration to remote work, companies can transition from traditional work environments to remote work efficiently. Currently, it is accepted practice for companies to subdivide individual job responsibilities into different tasks that can be completed outside the traditional workplace (Gubins et al., 2019). Technologies, such as laptops, smartphones, and Wi-Fi have facilitated remote work (Von Bergen et al., 2019). During the COVID-19 pandemic, governments mandated people to stay home, consequently forcing companies to adopt a remote work policy. It is likely that after the global pandemic ends, many people will continue to work remotely at least part of the time (Zhang et al., 2020).

Research has already shown changes in personal behavior from before the global pandemic started. These changes are related to technology. In one research study from China, 90\% of the respondents had more screen time than before the COVID-19 pandemic (Hu et al., 2020). For individuals and organizations, it is vital to understand how personality impacts the effectiveness of remote workers. The World Economic League Table stated that the trend of digitalization and remote work will continue. Working from home has become the norm for many white-collar workers because of technological changes (CEBR, 2020). 


\section{Theoretical Framework}

The present qualitative study was conducted by investigating personality traits of remote workers in China and used a phenomenological framework. Phenomenological research is well suited for this study because the goal is to understand people's lived experience with a phenomenon, consequently phenomenology can be used (Creswell et al., 2007; Gill, 2020). Specifically, a transcendental phenomenological framework was used to study how personality impacts remote workers in China. Participants in this research were individuals with remote work experience in China, and they were interviewed online using WeChat. All participants were informed that they were participating in research and signed a consent form allowing the interview to be recorded. Interviews were transcribed after they were completed using NVivo. All interviews were conducted in English.

For this study, the first step was to develop the core research questions; then, a review of the current literature was undertaken. The methodology selected for this research was qualitative data collection; specifically, a phenomenological approach was used. Research was conducted using the electronic collections available to students at California Southern University and Google

Scholar. The search engine Bing was sometimes used during the research process since the researcher lives in mainland China and Bing is a search engine that can consistently be accessed.

Subsequently, the review expanded to incorporate a systematic, critical review of previous studies related to remote work, trust, resilience, social exchange theory, leader member exchange, the 'Big Five,' Chinese culture, and personality. At its core, this study was about remote work and personality in China; however, without a deep understanding of Chinese culture, underlying aspects of this research could have been missed. Thus, time was spent defining essential concepts that are unique to China. While research conducted in Chinese was useful, all the research articles and sources for this study were published in English.

\section{Purpose of this Study}

This study aimed to facilitate understanding how people in China can better adapt to remote work. Personality traits can identify employees most suited for remote work. In addition to personality traits, self-determination theory was a core element of this research. Specifically, critical aspects were the universal psychological needs of autonomy, relatedness, and competency.

There are specific cultural factors that must be considered in this research. For example, the concept of face must be understood. Research has shown that face a central role in online user behaviors in China. Website operators can increase users' face by publishing the top contributors on a weekly, monthly, and annual basis (Wu \& Cheng, 2019). Face develops from social interactions, which are responsible for emotional experiences (Qi, 2011). Face is also influenced and shaped by self-examination, self-discipline, and self-improvement (Shi, 2019).

The researcher is from the United States, but he has lived and worked in China since 2012. During the COVID-19 lockdown from February 2020 until May 2020, the researcher experienced remote work. This experience and how it impacted the researcher was the genesis of the topic of this research. In the researcher's life, some of his colleagues thrived during remote work, while others struggled. The desire to explain this difference drove the researcher's interest in the topic of remote work. Furthermore, since the researcher lives in China, the idea of examining remote work from the perspective of Chinese workers appeared novel in the literature. The researcher neither offered any participants compensation for participation, nor received compensation from any source. 


\section{Method}

\section{Research Methodology}

To conduct phenomenological research, the best practice steps outlined by Moustakas (1994) were followed. These steps include having a topic and questions grounded with autobiographical and social significance, completing a comprehensive review of current literature, creating a set of questions to guide the interviews, holding and recording long-form person-toperson interviews, and analyzing data in a manner that facilitates textural and structural descriptions. The researcher followed these steps throughout the research process. Besides following the best practice methods for conducting phenomenological research, the researcher mindfully constructed the data collection and analysis mechanism. The reason for careful construction was to ensure as many extraneous variables were controlled as possible and demand characteristics were limited. Limiting demand characteristics helped increase validity by mitigating any need for participants to provide responses they thought the researcher wanted to hear. For the semi-structured portion of the interview, interview questions and key terms with definitions were sent to research participants ahead of time.

Moustakas (1994) also states researchers should provide instructions to co-researchers about the nature and purpose of the study and investigation. This step was not implemented because there were no co-researchers for this study. For narrative coding, coders meet to resolve discrepancies (McLean et al., 2020). Since this was an individual research problem, the researcher checked the coding done by NVivo to ensure accuracy. Audio recordings of each interview were listened to multiple times to determine and correct errors in coding.

At the center of this research project is an examination of what personality characteristics are found in productive and unproductive remote workers in China. To answer this sufficiently, a complete understanding of how individuals with remote work experience perceive and experience the phenomenon of remote work is essential. Moustakas (1994) listed characteristics for human scientific research. These characteristics are the research seeks to uncover the whole meaning and essence of the human experience, the research is qualitative rather than quantitative in its approach to behavior and experiences, the research participant is fully engaged, the research does not predict or determine casual relationships, and through careful, vivid, accurate recordings the lived experience is illuminated. The structure, aim, and implementation of this research project comply with the guidelines mentioned above. Compliance with the guidelines from Moustakas allowed the research process to avoid losing focus on the lived phenomenon of remote work in China.

\section{Participants}

Human participants were used to collect data. All participants for this study are from China, over 18 years old, speak English, and have professional remote work experience. Participation in this research was voluntary, and participants had the right to discontinue their participation at any time. Specific fields such as education, law, finance, international business, manufacturing, retail, graphic design, or any other professional work were not considered for inclusion. The research questions and phenomenon being studied were independent of a research participant's profession. Participants must have been working remotely, but their actual job was not studied; consequently, an individual's job was not a factor for participation. A participant's job title was also not a consideration for this research. Remote workers in management and non-management positions were both considered for this research. The broad inclusion of participations was imperative for 
this research because it allowed for triangulation of sources. Triangulation of sources is examining the consistency of various data sources inside the same method (Patton, 1999).

\section{Recruitment and Procedure}

All procedures and methods were approved by the author's university's institutional review board (IRB). All research participants were recruited from WeChat. This type of selection satisfied the requirements stated by Creswell (2018), a purposeful selection of participants to help the researcher understand the research question. Recruitment information was shared on WeChat. Recruitment information was also shared on LinkedIn; however, no participants were recruited from LinkedIn.

Participant selection does not need to be random or use a large sample size. A total of 7 participants were used for this study. The participants in this study came from several different professional fields. Participant A is a purchasing manager. Participant B works in logistics, and Participant C sells goods online. Participants D, E, F, and G are teachers who teach online. Participants D and E teach Chinese online. Participants F and G teach college entrance exam courses to Chinese students. The variety of industries represented by the participants was helpful because it provided a broad scope of remote work jobs and remote work experiences.

\section{Data Collection}

In this study, the sample size was seven people since qualitative research often utilizes a small sample size. Gill (2020) stated that phenomenology requires a small sample to reach saturation, and Giorgi (2017) also noted genuine reasons exist for using small samples. A small number of participants allows for all the data to be assessed. Guest et al. (2020) stated that the first five or six interviews produce most new information, and little new information is gained as the sample size nears 20 interviews. Their research also stated that using a base of four interviews is enough and that saturation can be assessed after six interviews (four in the base and two in the run). Although the sample size for this study is small by quantitative research standards, it is appropriate by qualitative research standards. Qualitative research is frequently rejected because the number of participants is considered too small even though adequate data exists. Quantitative analysis logic is inappropriately applied to qualitative analysis (Levitt, 2021). Qualitative methods and research often involve investigating only a few instances of a phenomenon in many different environments (Roald et al., 2021). Data saturation was reached after the seventh interview; consequently, data collection was discontinued at this point.

Interviews were done remotely using WeChat. All interviews were conducted one-on-one, using a semi-structured interview format. For the semi-structured portion of the interview, interview questions and key terms with definitions were sent to research participants ahead of time. The researcher develops an interview guide for a semi-structured interview that includes questions to focus on specific issues (Lukenchuk, 2017). In addition to meeting this best-practice requirement, the purpose of providing the research questions ahead of time was two-fold. The first reason was it allowed consistency between interviews by establishing core definitions of key vocabulary terms. Consistency between interviews helped eliminate the extraneous variable different questions being asked to the different participants; thus, inhibiting data analysis because of a lack of internal consistency.

Second, a semi-structured interview allowed research subjects to be fully prepared for the interview. Preparation was vital because all research participants were not native English speakers; consequently, providing interview questions before the interview helped decrease participants' 
anxiety and allowed them to ask questions before starting the interview. In situations where people share less linguistic and cultural background, clarification and interpretation of what is being said occur more often (Resnik, 2017). Providing the questions ahead of time helped offset the linguistic and cultural differences between the researcher and the participants. The extraneous variable of language was controlled and mitigated by providing questions ahead of time.

\section{Data Analysis}

After each interview was completed, the interviews were transcribed using NVivo because NVivo is one of the most popular qualitative data analysis software programs, and NVivo sets the standard for qualitative data analysis (Bonello \& Meehan, 2019). After NVivo transcribed the interview, the researcher reviewed the transcription line by line to identify obvious transcription errors. Then, the researcher went through the transcription again while listening to the audio recording of each interview. As subtle or obvious errors in transcription were discovered, the errors were corrected. Finally, an accurate transcript was produced, the transcript was imported into NVivo and coded. Coding is a key structure for organizing data in qualitative research (Williams \& Moser, 2019). It is essential to note here that no common language exists that crosses methods and traditions in qualitative research, so terms such as analysis or categorizing have the same meaning as coding (Levitt et al., 2017). Therefore, coding is the term used in this study. After NVivo coding was completed, the researcher printed the transcripts to check the coding done by NVivo. During this process, the researcher added codes manually. The reason for manually adding some codes is that nothing can take the place of manual coding (Lukenchuk, 2017).

The purpose of coding was to identify themes in the answers of participants. Moustakas (1994) referred to this process as clustering that involves, forming units into clusters or themes and removing overlapping statements. The codes and themes of each interview were compared to determine when participants no longer presented new information during the interview process. This methodology is supported by Levitt (2021), which states that qualitative researchers must consider the conditions and processes linked with the experience and the presentation of the phenomenon. Codes and themes allowed the researcher to clarify each research participant's lived experience and their unique experience with the phenomenon of remote work in China. The coding process allowed critical themes to be identified, codified, and analyzed to achieve the aim of the study (Williams \& Moser, 2019). Using NVivo software allows for more rigorous and transparent analysis (Bonello \& Meehan, 2019). However, the coding process is cyclical, and not all the first identified codes were part of the final list (Lukenchuk, 2017).

After coding was completed manually and through NVivo software, the researcher collapsed the codes into themes. Coding was done in two parts. The first part consisted of rereading the highlighted transcripts and codes to discover redundancies and overlaps, which were combined into overarching themes. Creating themes from the data is a common feature of qualitative research, and this method is widely used (Smith \& Firth, 2011).

\section{Results}

Remote work is a fascinating research area propelled to the forefront of people's conciseness because of the COVID-19 pandemic. The purpose of this research was to study how personality factors impact remote work in China because current literature does not adequately address this topic. The themes that emerged from the coding process allowed the researcher to draw several valuable conclusions. 
A total of four themes materialized during data analysis, namely, flexible time, responsibility, connectedness, and modesty. These themes were present across all interviews and were derived from consolidating codes. The themes of flexible time, responsibility, and connectedness provided a significant amount of information that the researcher used to answer the core research questions.

\section{Results for Research Question 1}

In the first research question, the goal was to understand the personality traits found in productive and unproductive remote workers. Responses for traits found in productive workers were isolated and compared in the interview transcripts. The most common responses centered around responsibility and self-discipline or self-control. These responses were given by Participants A, B, C, E, F, and G. The primary reason these traits were mentioned with such frequency was time management. Several participants spoke about needing responsibility and selfdiscipline as tools for managing one's time. Time management and self-discipline were the reasons that several participants stated they were productive remote workers. For participants who specifically addressed why remote workers are unproductive, time management, and self-discipline were identified as problems. The participants' specific descriptions emphasized that productive workers use time wisely, finish tasks on time, and are self-disciplined.

Another common response was related to extraversion. These responses referred to productive remote workers as being positive, energetic, talkative, social, and excited. Participant E used the words "energetic" and "excited" in several different answers. There was more of a divide between participants who work in education and those who work in business. Educators were more likely to list being positive, energetic, or talkative than people in business. Participant $\mathrm{C}$ sells items online, and she mentioned being social and talkative; however, these traits match a career in sales. Participant $\mathrm{G}$ frequently discussed passion and being energetic as foundational traits of productive remote workers. Participant A, who works as a purchasing manager, did not mention extraversion or traits that are associated with extraversion.

\section{Results for Research Question 2}

With the second research question, the researcher sought to discover how trust and cultural factors impact remote workers in China. Trust was a theme that was discovered after the coding process, elimination of redundant codes, and collapsing of codes were completed. Several participants discussed trust before being asked questions explicitly about Research Question 2. During the interview process, every participant was cooperative and open during the interview process. Some participants, such as Participant A, refused to discuss or acknowledge any cultural considerations. Participant D mentioned having prior work experience with some of her colleagues and her supervisor helped those individuals trust her as a remote worker. Participant $\mathrm{G}$ shared more personal antidotes and struggles than any other participant.

The most common responses for traits that are positively associated with developing trust were maintaining relationships, solving problems, and finishing work on time. Participants A, B, $\mathrm{C}, \mathrm{D}$, and $\mathrm{E}$ mentioned at least one of these traits. Participants $\mathrm{C}, \mathrm{D}$, and $\mathrm{F}$ mentioned communication.

Only Participant F discussed trust as being something developed mutually. This participant stated that mutual trust between her and her supervisor was very important. Mutual trust, in her experience, impacted her overall functioning at work. It is essential to note that Participant F and her current boss worked together at a previous company, so it is possible their pre-existing 
relationship impacted her response. All other participants answered questions about trust solely concerning what they needed to do. They did not address actions their supervisor or colleagues could take to impact trust.

\section{Results for Research Question 3}

The third research question's purpose was to determine how the psychological needs of autonomy, competency, and relatedness impact remote workers. Based on feedback from the participants, Research Question 3 was the most complex and challenging to understand. This question had multiple parts and used more academic language than other questions. Consequently, this question was broken down and discussed part by part during the interview process. The key terms generated before the interviews were beneficial for Research Question 3 because almost all the participants required an explanation of "autonomy," "competency," or "relatedness" before answering the question.

In every interview, autonomy was the first psychological need discussed. Participant B stated she felt autonomy is the most important thing for remote workers because without autonomy it is easy for remote workers to be lazy due to a lack of monitoring. Her response also linked directly to the theme of responsibility. Several participants stated that autonomy was valuable to them because it meant they could control their schedule. A connection exists between the psychological need for autonomy and the theme of flexible schedules for remote workers. Four different participants discussed the ability to control their schedule when asked about autonomy, and every participant discussed flexible schedules as a positive aspect of remote work.

In addition to having control over one's schedule, making decisions independently, and selecting which materials to use were mentioned by multiple participants. The ability to select materials was mentioned by Participants F and G. Both participants are online English teachers who help Chinese students prepare to take college entrance examinations. Making decisions independently was mentioned by Participant A and Participant D. Participant A works as a purchasing manager, and Participant $\mathrm{D}$ is an online Chinese teacher.

Competency was the second psychological need discussed during the interview process. The answers provided by participants were varied regarding how the need for competency impacts remote work. Some participants discussed competency as helping them to solve their problems.

An underlying sentiment existed during this part of the interview. Although only Participant E mentioned the feeling of confidence expressly, all participants' answers indicated that selfconfidence is a significant part of competency. The reason for this conclusion is that the most common response to questions about competency involved solving one's problems. The ability to solve problems with limited or no support is a core skill for remote workers because they do not have immediate, face-to-face contact with coworkers. Consequently, self-confidence allows remote workers to feel empowered when they face problems. Other responses also indicated that confidence is a major aspect of competency for remote workers. The ability to make decisions, to negotiate with supplies or students, and the ability to explain why lateness occurred were all examples provided by participants to demonstrate how they show competency.

Participants F and G discussed several ways they feel they lack competency as remote workers. Participant F spoke about not having coworkers to ask questions to and being unsure if she is doing a good job. Participant $G$ talked at length about her struggles with specific types of students and the negative impact these types of students have on her feeling of competency. The answers from these two participants demonstrated how critical competency is for remote workers. Each of these individuals has multiple years of remote work experience, but when the topic of 
competency was discussed, they immediately shared examples of how they lack competency. It must be noted that Participants F and $\mathrm{G}$ both teach college preparatory classes to Chinese students.

The final psychological need studied during the interview process was relatedness. Only Participants $\mathrm{A}$ and $\mathrm{C}$ did not share any negative aspects of remote work regarding relatedness. Participant A mentioned that contact and communication with others are important, but she did not express any feelings of loneliness. Participant $\mathrm{C}$ stated that the most important thing is having healthy relationships. She was also the only participant to state that people cannot work for just money. Participant $\mathrm{C}$ sells items online and views her interactions with others as a way to share her happiness.

Participants B, D, E, F, and G all shared that a lack of relatedness is a problem or concern for remote workers. Participant B shared that she felt lonely at home and had wanted to quit her job as a remote worker for the first couple of months because of the decrease in social interactions. She still goes to the office once or twice a month to maintain a sense of relatedness with her coworkers. No other participant mentioned wanting to quit being a remote worker. Participants $F$ and $\mathrm{G}$ both stated that relatedness is a problem for remote workers. Participant $\mathrm{G}$ also mentioned that face-to-face communication is precious and valuable. Participant D made an interesting point about being a remote worker and her previous professional experience. She mentioned that now she must find opportunities to communicate with others, whereas she had numerous opportunities to communicate and chat with other people before. All the research participants who are remote workers in the field of education expressed concerns about relatedness and remote work.

The analysis of these responses indicates an opportunity for future research related to remote work and the industry in which individuals work. Moreover, the responses about relatedness were beneficial to the core aspects of this research in another way: the participants did not mention COVID-19 or lockdowns as a reason they felt a lack of relatedness. This is noteworthy because it illustrates that the extraneous variable of working remotely as a byproduct of the pandemic was successfully controlled. Some participants occasionally mentioned the pandemic, but did so in passing and not as the reason the participants felt a certain way. The conclusions drawn from these responses appear to be influenced solely by being a remote worker in China.

Overall, the participants in this research study provided in-depth answers during the interview process. Their answers allowed the researcher to determine that autonomy is one of the most prominent positive aspects of remote work. Questions about which aspect of remote work participants enjoyed the most and a question about self-determination theory were the primary sources of information on autonomy. The need for competency is heavily influenced by confidence, and relatedness is negatively impacted by being a remote worker. Competency and relatedness were addressed directly, but participants at several points mentioned these two psychological needs during the interviews.

\section{Discussion}

This research study explored how personality factors impact remote workers in China. Phenomenology explores how people experience the world and use their experiences to describe and interpret human existence (Schatzki, 2017). Remote workers from China were interviewed so the researcher could understand their lived experiences. The purpose of understanding the lived experience of remote workers in China was to study how personality impacts these workers in China. Heidegger's ideas of people as being-in-the-world and being-in-the-world because of performing actions (Schatzki, 2017) were of paramount importance in this study.

The information provided by the research participants has practical implications for companies and remote workers. All the participants interviewed are currently employed as remote 
workers in China. From the perspective of the researcher, all participants were honest and forthright during the interview process. The interviewer did not suspect any feigning or any misrepresentation of facts from any interviewee. Demand characteristics were minimized so that participants would not attempt to provide a perceived correct answer to the interview questions. Bracketing and taking a dispassionate stance on thoughts shared by participants helped decrease any impact the researcher had on participants. Confidentiality, the right to discontinue participation in the interview, and the fact that participants had no prior relationships with the interviewer appeared to help participants answer as honestly as possible. As a result, each participant's lived experience can be drawn upon as a resource to help guide and influence companies that employ remote workers.

\section{Conclusion}

Remote work is a fascinating research area that was propelled to the forefront of people's consciousness because of the COVID-19 pandemic. Although many people had their first experience working remotely because of the pandemic, there are numerous people who were already remote workers. The purpose of this research was to study how personality factors impact remote work in China. People who started working remotely independently of the pandemic were the focus of this research because their lived experience as remote workers is different from the lived experience of someone working remotely due to quarantine or lockdown. Autonomy and, specifically, the ability to control one's schedule were noted as the most positive aspects of remote work. This conclusion is supported by previous literature that found that increased flexibility and autonomy are benefits of remote work (Mulki et al., 2009).

Modesty was an underlying personality facet for the people interviewed during the research process. Modesty has cultural roots and significance in Chinese culture. Previous personality research conducted on people from China supports the emergence of this theme. Bond et al. (2012) found three components of modest behavior in Chinese culture. These components are avoiding attention, effacing oneself, and enhancing others. These components were demonstrated by the participants in this research study. Participants F and G spent the most time effacing their abilities. In Chinese culture, people down-regulate their emotions due to the cultural emphasis on promoting social harmony and respect for societal roles (Hampton et al., 2021). Participants A and F were the most effusive in their praise for their boss.

Relatedness and feeling connected to others were common concerns among most of the research participants. Addressing these concerns is of paramount importance to individual remote workers and organizations that employ remote workers. In its most recent five-year economic plan, the Chinese government stated that employment is a pivotal part of safeguarding people's wellbeing (The 14th five-year plan for economic and social development of the People's Republic of China, 2021). This research coincides with the policies of the Chinese government because the research explored the personality factors are present in productive remote workers. Remote work is another avenue for employment, and when remote work is a suitable fit for a person's personality, then wellbeing through employment can be reached.

Remote work is a global occurrence, and understanding remote work from non-Western perspectives is essential. The researcher is hopeful that this study represents a significant contribution to the literature on the topic of remote work and, furthermore that it provided insights into the needs of remote workers in China. It is further hoped that this research will serve as a useful tool for future researchers, academics, and business leaders to use and that applying the conclusions of this research will improve remote work outcomes. 


\section{Limitations}

There are four limitations to this research. The first limitation is that all the research participants were female. Research participants were recruited voluntarily, and only females volunteered for participation. As a result of having only female participants, individuals who identify as male or as any gender other than female were not represented in this research. In mainland China, $51.24 \%$ of the population is male, and $48.76 \%$ is female (National Bureau of Statistics of China, 2021). If the study's population had been more diverse, it is possible codes such as family and child-rearing would not have appeared. Childcare and supporting a family were part of the rationale for becoming a remote worker for many participants. The homogenous participant group may impact this rationale. It is also possible that the theme of flexible time would have changed. This is because many of the participants enjoyed the time flexibility of remote work because they have child-rearing duties in addition to professional responsibilities. Future research on this topic might benefit from a research population that includes gender identities besides female.

The second limitation is that the research was conducted using English only. All the recruitment material was published in English, and all the interviews were conducted in English only. The use of English only served, without intent, as a point of exclusion. Only people from China who read and spoke English could participate in this research. Consequently, the total number of participants was decreased. Additionally, participants were forced to express themselves in English rather than in their native language. As previously stated, during the research process, steps were taken to mitigate language challenges.

The third limitation was that due to the COVID-19 pandemic and geographic limitations, all interviews relied on technology and a stable Internet connection. As a result, no face-to-face inperson interviews were conducted, which impacted rapport building. The researcher took every appropriate step to build rapport and create a safe environment, but this did not guarantee candid responses from interviewees.

The final limitation was the sample size. Phenomenological studies do not require large sample sizes; however, if more people were interviewed, that would have added depth to the research collected. With a small sample size, each participant's views had a more significant impact on the conclusions drawn from the research process.

\section{Suggestions for Future Research}

Here, some suggestions for future research are presented. First, the Big Five personality traits such as agreeableness and conscientiousness should be studied in a dual capacity. In this context, a dual capacity means studying remote workers outside of China and remote workers inside of China. The rationale is that the juxtaposition of remote workers outside of China and remote workers inside China will help future researchers to isolate cultural variables unique to people from China. The effort spent on this endeavor is worthwhile because of the rich cultural traditions found inside China. Meeting these cultural needs can help companies and employees. This research only focused on people from China, so no other group was studied.

The second suggestion for future research relates to the specific field or industry where remote workers are employed. Personality may impact remote workers in one field differently than remote workers in another. Separating remote workers by field would allow researchers to identify which personality traits impact remote workers in specific industries. The participants interviewed for this research demonstrated this difference, especially concerning the personality trait of extraversion. Future researchers could examine how personality impacts remote workers at a micro-level by isolating and looking at professional fields as separate entities. For example, 
individuals who work remotely in finance may be impacted by personality factors that are different from those that impact individuals working remotely in logistics or education. Different industries might desire a different mix of personality factors. The personality traits that make a great online teacher might not match the personality traits of an outstanding remote worker in logistics.

The final recommendation for future research is to explore remote workers, their personality traits, and gender. Future research could combine personality factors of specific jobs and a person's gender identity. Gender is another factor to consider because personality traits are not the same across males and females. Future research could benefit remote workers by delving deeper into how personality, gender, and job responsibilities impact each other. Thus, future research should focus on how the psychological needs of autonomy, competency, and relatedness manifest themselves differently across genders.

\section{References}

Bond, M, H., \& Lun, V, M, C., Chan, J., Chan, W, W, Y., \& Wong, D. (2012). Enacting modesty in Chinese culture: The joint contribution of personal characteristics and contextual features. Asian Journal of Social Psychology, 15, 14-25. https://doi.org/10.1111/j.1467839X.2011.01357.X

Bonello, M., \& Meehan, B. (2019). Transparency and coherence in a doctoral study case analysis: Reflecting on the use of NVivo within a 'framework' approach. The Qualitative Report, 24(3), 483-498. https://doi.org/10.46743/2160-3715/2019.3823

CEBR. (2020, December). World Economic League Table 2021 (12th edition). https://cebr.com/wp-content/uploads/2020/12/WELT-2021-final-23.12.pdf

Choi, S. (2019). Flexible work arrangements and employee retention: A longitudinal analysis of the federal workforces. Public Personnel Management, 49(3), 470-495. https://doi.org/10.1177/0091026019886340

Chong, A., Gordo, M., \& Gere, J. (2018). The influences of work and home interference and facilitation on job satisfaction. Journal of Personnel Psychology, 17(2), 94-101. https://doi.org/10.1027/1866-5888/a000202

Creswell, J. W. (2018). Research design: Qualitative, quantitative, and mixed methods approaches. SAGE Publications.

Creswell, J. W., Hanson, W. E., Clark Plano, V. L., \& Morales, A. (2007). Qualitative research designs: Selection and implementation. The Counseling Psychologist, 35, 236-264. https://doi.org/10.1177/0011000006287390

de Vos, D., Meijers, E., \& van Ham, M. (2018). Working from home and the willingness to accept a longer commute. The Annals of Regional Science, 61(2), 375-398. https://doi.org/10.1007/s00168-018-0873-6

Deci, E. L., \& Ryan, R. M. (2000). The "what" and "why" of goal pursuits: Human needs and the self-determination of behavior. Psychological Inquiry, 11(4), 227-268.

Gill, M. J. (2020). How can I study who you are? Methodological approaches to identify work research. In A. Brown (Ed.), The Oxford handbook of identities in organizations (pp. 295310). Oxford University Press.

Giorgi, A. (2017). A response to the attempted critique of the scientific phenomenological method. Journal of Phenomenological Psychology, 48, 83-144. https://doi.org/10.1163/1569162412341319

Gubins, S., Ommeren, J. V., \& Graaff, T. D. (2019). Does new information technology change commuting behavior? The Annals of Regional Science, 62(1), 187-210. https://doi.org/10.1007/s00168-018-0893-2 
Guest, G., Namey, E., \& Chen, M. (2020). A simple method to assess and report thematic saturation in qualitative research. Plos One, 15(5), 1-17. https://doi.org/10.1371/journal.pone.0232076

Hampton, R. S., Kwon, J. Y., \& Varnum, M. E. W. (2021). Variations in the regulation of affective neural responses across three cultures. Emotion, 21(2), 283-296. https://doi.org/10.1037/emo0000711

Hu, Z., Lin, X., Kaminga, A. C., \& Xu, H. (2020). Impact of the COVID-19 epidemic on lifestyle behaviors and their association with subjective well-being among the general population in mainland China: Cross-sectional study. Journal of Medical Internet Research, 22(8). https://doi.org/10.2196/21176

Jukka, M., Blomqvist, K., Li, P. P., \& Gan, C. (2017). Trust-distrust balance: Trust ambivalence in Sino-Western B2B relationships. Cross Cultural \& Strategic Management, 24(3), 482507. https://doi.org/10.1108/ccsm-01-2016-0019

Kim, M., \& Beehr, T., A. (2020). The long reach of the leader: Can empowering leadership at work result in enriched home lives? Journal of Occupational Health Psychology, 25(3), 203-213. https://dx.doi.org/10.1037/ocp0000177

Kuem, J., Khansa, L., \& Kim, S. S. (2020). Prominence and engagement: Different mechanisms regulating continuance and contribution in online communities. Journal of Management Information Systems, 37(1), 162-190. https://doi.org/10.1080/07421222.2019.1705510

Levitt, H. M. (2021). Qualitative generalization, not to the population but to the phenomenon: Reconceptualizing variation in qualitative research. Qualitative Psychology, 8(1), 95-110. https://dx.doi.org/10.1037/qup0000184

Levitt, H. M., Wertz, F. J., Motulsky, S. L., Morrow, S. L., \& Ponterotto, J. G. (2017). Recommendations for designing and reviewing qualitative research in psychology: Promoting methodological integrity. Qualitative Psychology, 4(1), 2-22. https://dx.doi.org/10.1037/qup0000082

Lukenchuk, A. (2017). Working with the data and presenting the findings. Counterpoints, 428, 101-117. https://www.jstor.org/stable/i40222682

McLean, K. C., Syed, M., Pasupathi, M., Adler, J. M., Dunlop, W. L., Drustrup, D., Fivush, R., Graci, M. E., Lilgendahl, J. P., Lodi-Smith, J., McAdams, D.P., \& Mccoy, T. (2020). The empirical structure of narrative identity: The initial big three. Journal of Personality and Social Psychology: Personality Processes and Individual Differences, 119(4), 920-944. https://doi.org/10.31234/osf.io/vu4qw

Morgeson, F. P., \& Humphrey, S. E. (2006). The work design questionnaire (WDQ): Developing and validating a comprehensive measure for assessing job design and the nature of work. Journal of Applied Psychology, 91(6), 1321-1339. https://doi.org/10.1037/00219010.91.6.1321

Moustakas, C. (1994). Phenomenological research methods. SAGE Publications.

Mulki, J., Bardhi, F., \& Lassk, F. G. (2009). Set up remote workers to thrive. MIT Sloan Management Review, 51(1), 63-69.

Nakrošienè, A., Bučiūnienė, I., \& Goštautaitė, B. (2019). Working from home: Characteristics and outcomes of telework. International Journal of Manpower, 40(1), 87-101. https://doi.org/10.1108/ijm-07-2017-0172

National Bureau of Statistics of China. (2021). Main Data of the Seventh National Population Census. http://www.stats.gov.cn/english

Nilles, J. M. (1991). Telecommuting and urban sprawl: Mitigator or inciter? Transportation, 18(4), 411-432. https://doi.org/10.1007/BF00186567 
Nilles, J. M. (1997). Telework: Enabling distributed organizations. Information Systems Management, 14(4), 7-14. https://doi.org/10.1080/10580539708907069

Patton, M., Q. (1999). Enhancing the quality and credibility of qualitative analysis. HSR: Health Services Research, 34(5 Pt 2), 1189-1208.

Potard, C., Henry, A., Boudoukha, A.-H., Courtois, R., Laurent, A., \& Lignier, B. (2020). Video game players' personality traits: An exploratory cluster approach to identifying gaming preferences. Psychology of Popular Media, 9(4), 499-512. https://doi.org/10.1037/ppm0000245

Qi, X. (2011). Face: A Chinese concept in global sociology. Journal of Sociology, 47(3), 279- 295. https://doi.org/10.1177/1440783311407692

Resnik, P. (2017). Metadiscourse in spoken interaction in ESL: A multilingual perspective. AAA: Arbeiten aus Anglistik und Amerikanistik, 17(2), 189-210.

Roald, T., Koppe, S., Jensen, T. B., Hansen, J. M., \& Levin, K. (2021). Why do we always generalize in qualitative research? Qualitative Psychology, 8(1), 69-81. https://dx.doi.org/10.1037/qup0000138

Schatzki, T. (2017). Pas de deux: Practice theory and phenomenology. Phänomenologische Forschungen, 2, 25-40. https://doi.org/10.28937/1000107734

Shi, Y. (2019). Contemporary contexts of Confucianism. Comparative Civilization Review, 80(80), 92-103. https://scholarsarchive.byu.edu/ccr/vol80/iss80/18/

Smith, J., \& Firth, J. (2011). Qualitative data analysis: Application of the framework approach. Nurse Researcher, 18(2), 52-62.

Smith, S. A., Patmos, A., \& Pitts, M. J. (2015). Communication and teleworking: A study of communication channel satisfaction, personality, and job satisfaction for teleworking employees. International Journal of Business Communication, 55(1), 44-68. https://doi.org/10.1177/2329488415589101

The 14th five-year plan for economic and social development of the People's Republic of China: 2021-2025. (2021). Central Compilation and Translation Press.

Unanue, W., Gómez, M. E., Cortez, D., Oyanedel, J. C., \& MendiburoSeguel, A. (2017). Revisiting the link between job satisfaction and life satisfaction: The role of basic psychological needs. Frontiers in Psychology, 8, 680. http://dx.doi.org/10.3389/fpsyg.2017.00680

Von Bergen, C. W., Bressler, M. S., \& Proctor, T. L. (2019) On the grid 24/7/365 and the right to disconnect. Employee Relations Law Journal, 45(2), 3-20.

Williams, M., \& Moser, T. (2019). The art of coding and thematic exploration in qualitative research. International Management Review, 15(1), 45-72.

Wu, Y., \& Cheng, Z. (2019). Formation of user stickiness in an online knowledge community in China. Social Behavior and Personality, 47(9), 1-14. https://doi.org/10.2224/sbp.8292

Zhang, S., Moeckel, R., Moreno, A. T., Shuai, B., \& Gao, J. (2020). A work-life conflict perspective on telework. Transportation Research Part A: Policy and Practice, 141, 5168. https://doi.org/10.1016/j.tra.2020.09.007 


\section{Notes on Contribution}

The research was conducted as the author's doctoral project for California Southern University under the guidance of Dr. Kelley Walters. Correspondence concerning this article should be addressed to Dr. Christopher Van Ham. Email: chrisvanham@yahoo.com; https://orcid.org/0000-0001-7643-3804

Manuscript received November 11, 2021

Final revision received January 18, 2022

Accepted January 20, 2022 